\title{
Prime Divisors of Palindromes
}

\author{
William D. BANKS \\ Department of Mathematics, University of Missouri \\ Columbia, MO 65211 USA \\ bbanks@math.missouri.edu \\ IGOR E. SHPARLINSKI \\ Department of Computing, Macquarie University \\ Sydney, NSW 2109, Australia \\ igor@ics.mq.edu.au
}

\begin{abstract}
In this paper, we study some divisibility properties of palindromic numbers in a fixed base $g \geq 2$. In particular, if $\mathcal{P}_{L}$ denotes the set of palindromes with precisely $L$ digits, we show that for any sufficiently large value of $L$ there exists a palindrome $n \in \mathcal{P}_{L}$ with at least $(\log \log n)^{1+o(1)}$ distinct prime divisors, and there exists a palindrome $n \in \mathcal{P}_{L}$ with a prime factor of size at least $(\log n)^{2+o(1)}$.
\end{abstract}

\section{Introduction}

For a fixed integer base $g \geq 2$, consider the base $g$ representation of an arbitrary natural number $n \in \mathbb{N}$ :

$$
n=\sum_{k=0}^{L-1} a_{k}(n) g^{k},
$$

where $a_{k}(n) \in\{0,1, \ldots, g-1\}$ for each $k=0,1, \ldots, L-1$, and the leading digit $a_{L-1}(n)$ is nonzero. The integer $n$ is said to be a palindrome if its digits satisfy the symmetry condition:

$$
a_{k}(n)=a_{L-1-k}(n), \quad k=0,1, \ldots, L-1 .
$$


It has recently been shown in [1] that almost all palindromes are composite.

For any $n \in \mathbb{N}$, the number $L$ in (1) is called the length of $n$; let $\mathcal{P}_{L} \subset \mathbb{N}$ denote the set of all palindromes of length $L$. In this paper, as in [1], we estimate exponential sums of the form

$$
S_{q}(L ; c)=\sum_{n \in \mathcal{P}_{L}} \mathbf{e}_{q}(c n),
$$

where as usual $\mathbf{e}_{q}(x)=\exp (2 \pi i x / q)$ for all $x \in \mathbb{R}$. Using these estimates, we show that for all sufficiently large values of $L$, there exists a palindrome $n \in \mathcal{P}_{L}$ with at least $(\log \log n)^{1+o(1)}$ distinct prime divisors, and there exists a palindrome $n \in \mathcal{P}_{L}$ with a prime factor of size at least $(\log n)^{2+o(1)}$.

Throughout the paper, all constants defined either explicitly or implicitly via the symbols $O, \Omega, \ll$ and $\gg$ may depend on $g$ but are absolute otherwise. We recall that, as usual, the following statements are equivalent: $A=O(B)$, $B=\Omega(A), A \ll B$, and $B \gg A$. We also write $A \asymp B$ to indicate that $B \ll A \ll B$.

Acknowledgements. The authors wish to thank Florian Luca for some useful conversations. Most of this work was done during a visit by the authors to the Universidad de Cantabria; the hospitality and support of this institution is gratefully acknowledged. W. B. was supported in part by NSF grant DMS-0070628, and I. S. was supported in part by ARC grant DP0211459.

\section{Preliminary Results}

For every natural number $q$ with $\operatorname{gcd}(q, g)=1$, we denote by $t_{q}$ the order of $g$ in the multiplicative group modulo $q$. For arbitrary integers $a, b, K$ with $K \geq 1$ we consider the exponential sums

$$
T_{q}(a, b)=\sum_{k=1}^{t_{q}} \mathbf{e}_{q}\left(a g^{k}+b g^{-k}\right) \quad \text { and } \quad T_{q}(K ; a, b)=\sum_{k=1}^{K} \mathbf{e}_{q}\left(a g^{k}+b g^{-k}\right),
$$

where the inversion $g^{-k}$ is taken in the residue ring $\mathbb{Z}_{q}$.

Lemma 1. Let $\mathcal{S}$ be a set of primes coprime to $g$, with $\operatorname{gcd}\left(t_{p_{1}}, t_{p_{2}}\right)=1$ for all distinct $p_{1}, p_{2} \in \mathcal{S}$. Then for the integer $q=\prod_{p \in \mathcal{S}} p$ one has

$$
T_{q}(a, b)=\prod_{p \in \mathcal{S}} T_{p}(a, b) .
$$


Proof. Consider the Kloosterman sums

$$
K_{\chi}(a, b ; q)=\sum_{\substack{1 \leq c \leq q \\ \operatorname{gcd}(c, q)=1}} \chi(c) \mathbf{e}_{q}(a c+b \bar{c})
$$

as $\chi$ varies over the multiplicative characters of $\mathbb{Z}_{q}^{*}$. Denoting by $X_{q}$ the group of all such characters for which $\chi(g)=1$, as in the proof of Lemma 2.1 of [1] one has

$$
T_{q}(a, b)=\frac{t_{q}}{\varphi(q)} \sum_{\chi \in X_{q}} K_{\chi}(a, b ; q)
$$

Because of the assumed property of the set $\mathcal{S}$, we see that $t_{q}=\prod_{p \in \mathcal{S}} t_{p}$, and therefore

$$
\# X_{q}=\frac{\varphi(q)}{t_{q}}=\prod_{p \in \mathcal{S}} \frac{\varphi(p)}{t_{p}}=\prod_{p \in \mathcal{S}} \# X_{p} .
$$

By duality theory, it follows that $X_{q}$ is the direct product of the groups $\left\{X_{p}: p \in \mathcal{S}\right\}$, hence every character $\chi \in X_{q}$ has a unique decomposition

$$
\chi=\prod_{p \in \mathcal{S}} \chi_{p}
$$

where $\chi_{p} \in X_{p}$ for each $p \in \mathcal{S}$. By the well known multiplicative property of Kloosterman sums,

$$
K_{\chi}(a, b ; q)=\prod_{p \in \mathcal{S}} K_{\chi_{p}}(a, b ; p)
$$

and therefore

$$
T_{q}(a, b)=\frac{t_{q}}{\varphi(q)} \sum_{\chi \in X_{q}} \prod_{p \in \mathcal{S}} K_{\chi_{p}}(a, b ; p)=\prod_{p \in \mathcal{S}} \frac{t_{p}}{\varphi(p)} \sum_{\chi_{p} \in X_{p}} K_{\chi_{p}}(a, b ; q) .
$$

The result follows.

Lemma 2. Let $\mathcal{S}$ be a set of primes $p$ such that $p \geq z, p \equiv 3(\bmod 4)$, $\operatorname{gcd}(p, g(g-1))=1$, and $t_{p}=\Omega\left(\log ^{2} p\right)$ for every $p \in \mathcal{S}$. Suppose that $\operatorname{gcd}\left(t_{p_{1}}, t_{p_{2}}\right) \leq 2$ for all distinct $p_{1}, p_{2} \in \mathcal{S}$. If $z$ is sufficiently large, then for some absolute constant $A>0$ and all $a, b \in \mathbb{Z}$ one has

$$
\left|T_{q}(a, b)\right| \leq t_{q} \prod_{\substack{p \in \mathcal{S} \\ \operatorname{gcd}(a, b, p)=1}}\left(1-\frac{A}{\log p(\log \log p)^{5}}\right)
$$

where $q=\prod_{p \in \mathcal{S}} p$. 
Proof. If $t_{q}$ is odd, then $\operatorname{gcd}\left(t_{p_{1}}, t_{p_{2}}\right)=1$ for all distinct $p_{1}, p_{2} \in \mathcal{S}$, thus

$$
t_{q}=\prod_{p \in \mathcal{S}} t_{p}
$$

By Lemma 1, we also have

$$
T_{q}(a, b)=\prod_{p \in \mathcal{S}} T_{p}(a, b) .
$$

Moreover,

$$
T_{p}(a, b)=\frac{t_{p}}{p-1} \sum_{x \in \mathbb{Z}_{p}^{*}} \mathbf{e}_{p}\left(a x^{(p-1) / t_{p}}+b x^{-(p-1) / t_{p}}\right)
$$

for all $p \in \mathcal{S}$. If $\operatorname{gcd}(a, b, p)=1$, then since $t_{p}=\Omega\left(\log ^{2} p\right)$, Theorem 1.1 of [2] implies that the estimate

$$
\left|\sum_{x \in \mathbb{Z}_{p}^{*}} \mathbf{e}_{p}\left(a x^{(p-1) / t_{p}}+b x^{-(p-1) / t_{p}}\right)\right| \leq(p-1)\left(1-\frac{A}{\log p(\log \log p)^{5}}\right)
$$

holds for some absolute constant $A>0$ provided that $z$ is large enough. On the other hand, $T_{p}(a, b)=t_{p}$ if $\operatorname{gcd}(a, b, p)=p$. This completes the proof in the case that $t_{q}$ is odd.

If $t_{q}$ is even, then the multiplicative order of $g^{2}$ modulo $q$ is $\tau_{q}=t_{q} / 2$, and for each $p \in \mathcal{S}$ the multiplicative order of $g^{2}$ modulo $p$ is $\tau_{p}=t_{p} / 2$ or $\tau_{p}=t_{p}$ according to whether $t_{p}$ is even or odd, respectively. Since each prime $p \in \mathcal{S}$ is congruent to $3(\bmod 4)$, it follows that $\tau_{p}$ is odd, and we have

$$
\tau_{q}=\prod_{p \in \mathcal{S}} \tau_{p} .
$$

We now write

$$
T_{q}(a, b)=\sum_{k=1}^{\tau_{q}} \mathbf{e}_{q}\left(a f^{k}+b f^{-k}\right)+\sum_{k=1}^{\tau_{q}} \mathbf{e}_{q}\left(a g f^{k}+b g^{-1} f^{-k}\right)
$$

where $f=g^{2}$. Noting that $\tau_{p}=\Omega\left(\log ^{2} p\right)$ for all $p \in \S$, we can apply the preceding argument to both of these sums (with $g$ replaced by $g^{2}$ ), and we derive the stated result in the case that $t_{q}$ is even. 
Lemma 3. If $y$ is sufficiently large, there is a set $\mathcal{S} \in\left[y(\log y)^{-2}, y\right]$ of primes $p$ with $p \equiv 3(\bmod 4)$ and $\operatorname{gcd}\left(p, g\left(g^{2}-1\right)\right)=1$, of cardinality at least $\# \mathcal{S}=\Omega\left(y^{1 / 4}(\log y)^{-2}\right)$, such that $\operatorname{gcd}\left(t_{p_{1}}, t_{p_{2}}\right) \leq 2$ for any distinct $p_{1}, p_{2} \in \mathcal{S}$, and $t_{p} \geq p^{1 / 4}$ for all $p \in \mathcal{S}$.

Proof. According to Lemma 1 of [3] (taking $k=1, u=3$ and $v=16$ in that lemma), for every sufficiently large value of $y$ there are at least $\Omega\left(y / \log ^{2} y\right)$ primes $p \leq y$ with $p \equiv 3(\bmod 16)$ such that either $p=2 r_{1} r_{2}+1$ where $r_{1}, r_{2} \geq y^{1 / 4}$ are primes, or $p=2 r_{0}+1$ where $r_{0}$ is a prime. Clearly, the interval $\left[y(\log y)^{-2}, y\right]$ also contains a set $\mathcal{L}$ of $\Omega\left(y / \log ^{2} y\right)$ such primes. Note that for $y$ large enough, we have that $p \nmid g\left(g^{2}-1\right)$ for each $p \in \mathcal{L}$.

Take the smallest such prime $p_{1} \in \mathcal{L}$ and put it into the set $\mathcal{S}$. Next, remove all primes $p \in \mathcal{L}$ for which $\operatorname{gcd}\left(p-1, p_{1}-1\right)>2$; since this condition implies that $\operatorname{gcd}\left(p-1, p_{1}-1\right) \geq y^{1 / 4}$, we remove at most $O\left(y^{3 / 4}\right)$ such primes at this step. Now take the smallest remaining prime $p_{2} \in \mathcal{L}$ and add it to $\mathcal{S}$, then remove the $O\left(y^{3 / 4}\right)$ primes $p \in \mathcal{L}$ for which $\operatorname{gcd}\left(p-1, p_{2}-1\right)>2$. Continuing in this manner, we eventually put $\Omega\left(\# \mathcal{L} y^{-3 / 4}\right)=\Omega\left(y^{1 / 4}(\log y)^{-2}\right)$ primes into the set $\mathcal{S}$. Noting that each $t_{p}>2$ and $t_{p} \mid p-1$, it follows that $t_{p} \geq y^{1 / 4} \geq p^{1 / 4}$ for every $p \in \mathcal{S}$.

We also need the following bound for incomplete sums:

Lemma 4. For any prime $p$ with $\operatorname{gcd}(p, g)=1$ and any natural number $K \leq t_{p}$, the following bound holds:

$$
\max _{\operatorname{gcd}(a, b, p)=1}\left|T_{p}(K ; a, b)\right| \ll p^{1 / 2} \log p .
$$

Proof. It is easy to see that for any $h=0, \ldots, t_{p}$,

$$
\sum_{k=1}^{t_{p}} \mathbf{e}_{p}\left(a g^{k}+b g^{-k}\right) \mathbf{e}_{t_{p}}(h k)=\frac{t_{p}}{p-1} \sum_{x \in \mathbb{F}_{p}^{*}} \mathbf{e}_{p}\left(a x^{(p-1) / t_{p}}+b x^{-(p-1) / t_{p}}\right) \chi(x)
$$

where $\chi(x)$ is a certain multiplicative character on $\mathbb{F}_{p}^{*}$. Applying the Weil bound to the last sum (see Example 12 in Appendix 5 of [6]; also Theorem 3 of Chapter 6 in [4], and Theorem 5.41 and the comments to Chapter 5 in [5]), we derive that

$$
\sum_{k=1}^{t_{p}} \mathbf{e}_{p}\left(a g^{k}+b g^{-k}\right) \mathbf{e}_{t_{p}}(h k) \ll p^{1 / 2}
$$

Now using the standard reduction from complete sums to incomplete ones, we obtain the desired result. 
A relation between the sums $S_{q}(L ; c)$ and $T_{q}(K ; a, b)$ has been found in [1] which we now present in a slightly modified form.

Lemma 5. Let $K=\lfloor L / 2\rfloor$. Then

$$
\left|S_{q}(L ; c)\right| \leq g^{2}\left(g^{2}-1+\frac{1}{K}\left|T_{q}\left(K ; c, c g^{L-1}\right)\right|\right)^{K / 2} .
$$

Proof. As in the proof of Lemma 3.1 of [1] we have

$$
\left|S_{q}(L ; c)\right| \leq g^{2} \prod_{k=1}^{K}\left|\sum_{a=0}^{g-1} \mathbf{e}_{q}\left(a c\left(g^{k}+g^{L-1-k}\right)\right)\right| .
$$

Then, using the arithmetic-geometric mean inequality, we derive that

$$
\begin{aligned}
\left|S_{q}(L ; c)\right| & \leq g^{2}\left(\frac{1}{K} \sum_{k=1}^{K}\left|\sum_{a, b=0}^{g-1} \mathbf{e}_{q}\left(a c\left(g^{k}+g^{L-1-k}\right)\right)\right|^{2}\right)^{K / 2} \\
& =g^{2}\left(\frac{1}{K} \sum_{a, b=0}^{g-1} \sum_{k=1}^{K} \mathbf{e}_{q}\left(c(a-b)\left(g^{k}+g^{L-1-k}\right)\right)\right)^{K / 2}
\end{aligned}
$$

Estimating each inner sum trivially as $K$ for all $a$ and $b$ except for $a=1$, $b=0$, we obtain the desired statement.

\section{Exponential Sums with Palindromes}

Theorem 6. There exists a constant $B>0$ such that for all sufficiently large values of $L$ and any prime $p \leq L^{2} / \log ^{4} L$ such that $\operatorname{gcd}(p, g(g-1))=1$, the following bound holds:

$$
\max _{\operatorname{gcd}(c, p)=1}\left|S_{p}(L ; c)\right| \leq \# \mathcal{P}_{L} \exp \left(-L / \log p(\log \log p)^{B}\right) .
$$

Proof. Taking $K=\lfloor L / 2\rfloor$, we have by Lemma 5 :

$$
\left|S_{p}(L ; c)\right| \leq g^{2}\left(g^{2}-1+\frac{1}{K}\left|T_{p}\left(K ; c, c g^{L-1}\right)\right|\right)^{K / 2}
$$


Suppose that $\operatorname{gcd}(c, p)=1$. Let us write $K=Q t_{p}+R$ where $Q \geq 0$ and $0 \leq R<t_{p}$.

Let us first consider the case $K \geq t_{p}$. Since $p \mid\left(g^{t_{p}}-1\right)$, it is clear that $t_{p}=\Omega(\log p)$; using Theorem 1.1 of [2] as in the proof of Lemma 2, it follows that for all sufficiently large primes $p$,

$$
\left|T_{p}\left(c, c g^{L-1}\right)\right| \leq t_{p}\left(1-\frac{1}{\log p(\log \log p)^{C_{0}}}\right)
$$

for some constant $C_{0}>0$. Moreover, for any prime $p$ coprime to $g(g-1)$, it is clear that $t_{p} \neq 1$ and that

$$
\left|T_{p}\left(c, c g^{L-1}\right)\right|<t_{p}
$$

Therefore, adjusting the value of $C_{0}$ if necessary, we see that the bound (3) holds for every prime $p$ such that $\operatorname{gcd}(p, g(g-1))=1$. Thus, in the case that $K \geq t_{p}$ we have

$$
\begin{aligned}
\left|T_{p}\left(K ; c, c g^{L-1}\right)\right| & =Q\left|T_{p}\left(c, c g^{L-1}\right)\right|+\left|T_{p}\left(R ; c, c g^{L-1}\right)\right| \\
& \leq Q t_{p}\left(1-\frac{1}{\log p(\log \log p)^{C_{0}}}\right)+R \\
& =K-\frac{Q t_{p}}{\log p(\log \log p)^{C_{0}}} \leq K\left(1-\frac{1}{2 \log p(\log \log p)^{C_{0}}}\right) .
\end{aligned}
$$

When $K<t_{p}$ we apply Lemma 4 to deduce that

$$
\left|T_{p}\left(K ; c, c g^{L-1}\right)\right| \ll p^{1 / 2} \log p \ll K(\log p)^{-1},
$$

since $K \gg L \geq p^{1 / 2}(\log p)^{2}$. Thus, in this case, we have a much stronger bound.

Therefore, for sufficiently large $p$,

$$
\begin{aligned}
g^{2}-1+\frac{1}{K}\left|T_{p}\left(K ; c, c g^{L-1}\right)\right| & \leq g^{2}-\frac{1}{2 \log p(\log \log p)^{C_{0}}} \\
& \leq g^{2} \exp \left(-\frac{1}{2 g^{2} \log p(\log \log p)^{C_{0}}}\right)
\end{aligned}
$$

Using this estimate in (2) together with the obvious relation $\# \mathcal{P}_{L} \asymp g^{L / 2}$, we derive the stated result. 


\section{Congruences with Palindromes}

Let us denote

$$
\mathcal{P}_{L}(q)=\left\{n \in \mathcal{P}_{L}: n \equiv 0 \quad(\bmod q)\right\} .
$$

Proposition 4.2 of [1] asserts that if $\operatorname{gcd}\left(q, g\left(g^{2}-1\right)\right)=1$, then for $L \geq$ $10+2 q^{2} \log q$ the following asymptotic formula holds:

$$
\# \mathcal{P}_{L}(q)=\frac{\# \mathcal{P}_{L}}{q}+O\left(\frac{\# \mathcal{P}_{L}}{q} \exp \left(-\frac{L}{2 q^{2}}\right)\right)
$$

Here we obtain a nontrivial bound on $\# \mathcal{P}_{L}(q)$ without any restrictions on the size or the arithmetic structure of $q$.

Theorem 7. For all positive integers $L$ and $q$, the following bound holds:

$$
\# \mathcal{P}_{L}(q) \ll \frac{\# \mathcal{P}_{L}}{q^{1 / 2}}
$$

Proof. Let $r$ be the largest integer for which $r \equiv L(\bmod 2)$ and $g^{r} \leq q$. Clearly, $g^{r} \gg q$. We observe that every palindrome $n \in \mathcal{P}_{L}$ can be expressed in the form

$$
n=g^{(L+r) / 2} k_{1}+g^{(L-r) / 2} m+k_{2}
$$

where $k_{1}, k_{2}<g^{(L-r) / 2}, g^{(L-r) / 2} k_{1}+k_{2}$ is a palindrome of length $L-r$, and $m<g^{r}$. Note that for each choice of $k_{2}$, the value of $k_{1}$ is uniquely determined by the palindromy condition.

Let $d=\operatorname{gcd}(q, g)$. If $n \in \mathcal{P}_{L}$ is divisible by $q$, then $d \mid k_{2}$; since $k_{2} \neq 0$ there are at most $g^{(L-r) / 2} / d$ choices for $k_{2}$. Since $g^{r} \leq q$, it follows that for each choice of $k_{2}$ there are at most $d$ values of $m<g^{r}$ such that the congruence $g^{(L+r) / 2} k_{1}+g^{(L-r) / 2} m+k_{2} \equiv 0(\bmod q)$ holds. Therefore, $\# \mathcal{P}_{L}(q) \leq g^{(L-r) / 2} \ll \# \mathcal{P}_{L} q^{-1 / 2}$.

\section{Prime Divisors of Palindromes}

Let $\omega(n)$ denote the number of distinct prime divisors of an integer $n \geq 2$.

Theorem 8. For all sufficiently large L, there is a palindrome $n$ whose length is $L$ and for which

$$
\omega(n)=\Omega\left(\frac{\log \log n}{\log \log \log n}\right) .
$$


Proof. Define $y$ by the equation

$$
2 C_{1} y^{1 / 4}(\log y)^{-1}=\log L
$$

where $C_{1}$ is the constant implied by the $\Omega$-symbol in Lemma 3 , and let $\mathcal{S}$ be a set of primes of cardinality $\# \mathcal{S}=\left\lfloor C_{1} y^{1 / 4}(\log y)^{-2}\right\rfloor$ with the properties stated in that lemma. Putting

$$
q=\prod_{p \in \mathcal{S}} p
$$

by Lemma 2 we see that

$$
\max _{\operatorname{gcd}(a, b, q)<q}\left|T_{q}(a, b)\right| \leq t_{q}\left(1-\frac{C_{2}}{\log y(\log \log y)^{5}}\right)
$$

for some constant $C_{2}>0$ provided that $L$ is large enough. In particular, supposing that $\operatorname{gcd}(c, q)=1$, we obtain the estimate

$$
\left|T_{q}\left(c, c g^{L-1}\right)\right| \leq t_{q}\left(1-\frac{C_{2}}{\log y(\log \log y)^{5}}\right)
$$

since $\operatorname{gcd}(g, q)=1$ for sufficiently large $L$. Taking $K=\lfloor L / 2\rfloor$, we have by Lemma 5:

$$
\left|S_{q}(L ; c)\right| \leq g^{2}\left(g^{2}-1+\frac{1}{K}\left|T_{q}\left(K ; c, c g^{L-1}\right)\right|\right)^{K / 2} .
$$

As in the proof of Theorem 6 , we now write $K=Q t_{q}+R$ with integers $Q \geq 0$ and $0 \leq R<t_{q}$. Because $K=\lfloor L / 2\rfloor \geq\left(t_{q}^{2}-1\right) / 2 \geq t_{q}$ we have $Q \geq 1$. Thus, provided that $L$ is large enough, using (4) we derive

$$
\begin{aligned}
\left|T_{q}(K ; c, c)\right| & =Q\left|T_{q}(c, c)\right|+\left|T_{q}(R ; c, c)\right| \\
& \leq Q t_{q}\left(1-\frac{C_{2}}{\log y(\log \log y)^{5}}\right)+R \\
& =K-\frac{C_{2} Q t_{q}}{\log y(\log \log y)^{5}} \leq K\left(-\frac{C_{2}}{2 \log y(\log \log y)^{5}}\right),
\end{aligned}
$$

since $Q t_{q} \geq Q>R$.

Applying this to (5), it follows that

$$
\left|S_{q}(L ; c)\right| \leq \# \mathcal{P}_{L} \exp \left(-C_{4} L / \log y(\log \log y)^{5}\right)
$$


for some constant $C_{4}>0$, provided that $\operatorname{gcd}(c, q)<q$ and $L$ is sufficiently large.

Now let us denote

$$
\mathcal{P}_{L}(q, a)=\left\{n \in \mathcal{P}_{L}: n \equiv a \quad(\bmod q)\right\} .
$$

By the same arguments given in the proof of Proposition 4.2 of [1], it is easily shown that the preceding estimate implies

$$
\# \mathcal{P}_{L}(q, a)=\frac{\# \mathcal{P}_{L}}{q}+O\left(\# \mathcal{P}_{L} \exp \left(-C_{4} L / \log y(\log \log y)^{5}\right)\right)
$$

In particular $\mathcal{P}_{L}(q, 0)>0$ for sufficiently large $L$. Taking any $n \in \mathcal{P}_{L}(q, 0)$ we obtain $\omega(n) \geq \omega(q) \geq \# \mathcal{S}=\Omega\left(y^{1 / 4}(\log y)^{-2}\right)$, and since $L \asymp \log n$ the result follows.

Theorem 9. There is a constant $C>0$ such that for all sufficiently large $L$

$$
\prod_{\substack{p \leq L^{2}(\log L)^{-C} \\ \operatorname{gcd}(p, g(g-1))=1}} p \mid \prod_{n \in \mathcal{P}_{L}} n
$$

Proof. Repeating the same arguments as in the proof of Proposition 4.1 of [1], we derive from Theorem 6 that

$$
\# \mathcal{P}_{L}(p, a)=\frac{\# \mathcal{P}_{L}}{p}+O\left(\# \mathcal{P}_{L} \exp \left(-L / 2 \log p(\log \log p)^{B}\right)\right)
$$

where, $B$ is defined in Theorem 6 and as before, $\mathcal{P}_{L}(p, a)$ is defined by (6). In particular, $\# \mathcal{P}_{L}(p, 0)>0$ provided that $L$ is large enough.

Theorem 9 immediately implies that

$$
\omega\left(\prod_{n \in \mathcal{P}_{L}} n\right)=\Omega\left(\frac{L^{2}}{(\log L)^{C}}\right) .
$$

We now use Theorem 7 to derive a more precise result.

Theorem 10. For all sufficiently large $L$,

$$
\omega\left(\prod_{n \in \mathcal{P}_{L}} n\right)=\Omega\left(\frac{L^{2}}{(\log L)^{2}}\right)
$$


Proof. Let

$$
W=\prod_{n \in \mathcal{P}_{L}} n, \quad s=\omega(W) .
$$

For each prime $p$, we denote by $r_{p}$ the exact power of $p$ dividing $W$; then

$$
\prod_{n \in \mathcal{P}_{L}} n=\prod_{p \mid W} p^{r_{p}}
$$

and this implies that

$$
r_{p}=\sum_{\alpha=1}^{\infty} \# \mathcal{P}_{L}\left(p^{\alpha}\right) .
$$

By Theorem 7 we have the estimate

$$
r_{p} \ll \# \mathcal{P}_{L} \sum_{\alpha=1}^{\infty} p^{-\alpha / 2} \ll \frac{\# \mathcal{P}_{L}}{p^{1 / 2}}
$$

thus,

$$
\# \mathcal{P}_{L} \sum_{p \mid W} \frac{\log p}{p^{1 / 2}} \gg \sum_{p \mid W} r_{p} \log p=\log W \gg L g^{L} .
$$

Denoting by $p_{j}$ the $j$-th prime number, we obtain

$$
L \ll \sum_{p \mid W} \frac{\log p}{p^{1 / 2}} \leq \sum_{j=1}^{s} \frac{\log p_{j}}{p_{j}^{1 / 2}} \ll(s \log s)^{1 / 2}
$$

which finishes the proof.

\section{Remarks}

It is an open question (posed in [1]) as to whether there exist infinitely many prime palindromes in a given base $g \geq 2$, and the solution appears to be quite difficult. Indeed, since the collection of palindromes in any base forms a set as thin as that of the square numbers, this question is likely to be as difficult as that of showing the existence of infinitely many primes of the form $p=n^{2}+1$. At the present time, however, even the question as to whether there exist infinitely squarefree palindromes remains open. 


\section{References}

[1] W. Banks, D. Hart and M. Sakata, 'Almost all palindromes are composite', to appear in Math. Res. Lett.

[2] T. Cochrane, C. Pinner and J. Rosenhouse, 'Bounds on exponential sums and the polynomial Waring problem mod p', J. London Math. Soc. (2) 67 (2003) no. 2, 319-336.

[3] D. R. Heath-Brown, 'Artin's conjecture for primitive roots', Quart. J. Math., 37 (1986), 27-38.

[4] W.-C. W. Li, Number theory with applications, World Scientific, Singapore, 1996.

[5] R. Lidl and H. Niederreiter, Finite fields, Cambridge University Press, Cambridge, 1997.

[6] A. Weil, Basic number theory, Springer-Verlag, New York, 1974. 